\title{
L'automobile de demain : une histoire de générations déçues
}

\section{Gijs Mom}

\section{(2) OpenEdition}

1 Journals

\section{Édition électronique}

URL : https://journals.openedition.org/artefact/7613

DOI : 10.4000/artefact.7613

ISSN : 2606-9245

Éditeur:

Association Artefact. Techniques histoire et sciences humaines, Presses universitaires du Midi

\section{Édition imprimée}

Date de publication : 18 juin 2015

Pagination : 15-25

ISBN : 978-2-271-08155-1

ISSN : 2273-0753

\section{Référence électronique}

Gijs Mom, «L'automobile de demain : une histoire de générations déçues », Artefact [En ligne], HS 01 । 2015, mis en ligne le 30 avril 2021, consulté le 05 mai 2021. URL : http://journals.openedition.org/ artefact/7613; DOI : https://doi.org/10.4000/artefact.7613

\section{cc) (†)}

Artefact, Techniques, histoire et sciences humaines est mise à disposition selon les termes de la Licence Creative Commons Attribution - Pas d'Utilisation Commerciale - Pas de Modification 4.0 International. 


\section{L'automobile de demain : une histoire de générations déçues}

Gijs MoM*

\section{Résumé}

Cet article est la première synthèse d'un ouvrage de 400 pages publié en 2004 (note 1). L'argumentation se fonde d'abord sur la déconstruction d'explications généralement apportées par des ingénieurs pour comprendre l'échec du véhicule électrique. Autrement dit, si nous l'avions choisi, nous aurions pu rouler à l'électricité sans problème. Face aux arguments techniques, l'auteur avance une hypothèse culturelle selon laquelle la voiture électrique est en fait un succès dans la mesure où elle est parvenue à stimuler la voiture à pétrole dans ses perfectionnements techniques. En l'absence d'analyse plus poussée sur la seconde moitié du xx siècle, il convient de s'interroger sur notre changement d'opinion sur la faisabilité de la voiture électrique. En avons-nous réellement changé? Devons-nous réellement le faire?

Mots-clés : automobilisme, culture automobile, technique automobile, voiture électrique.

\section{Abstract}

This is the first full summary of the argumentation of an original 400 pages study (note 1) published in 2004. Methodologically, the argument is based first on a successive unravelling of explanations brought forward by most engineers to know why the electric vehicles was never successful. In other words, if people had wanted to, they could have driven electrically without any problem. Then, instead of technical arguments, a cultural explanation is proposed, and it is argued that the electric vehicles did not fail but were very successful, in pushing the mainstream, combustion car to ever higher levels of sustaina-

* Gijs Mom (Technische Universiteit Eindhoven, Pays-Bas) est historien, spécialiste de l'histoire des mobilités. Il a notamment travaillé sur l'histoire culturelle de l'automobile, tout en contribuant à restructurer le champ de l'histoire des transports en un champ nouveau, celui de l'histoire des mobilités. Président-fondateur de l'association internationale T2M, il est actuellement éditeur de la revue Transfers qu'il a également créée. Dernière publication: Atlantic automobilism: the emergence and persistence of the car, 1895-1940, New York, Berghahn Books, 2014. 
bility. Although a thorough analysis of the second half of the xxth century is still lacking, it is necessary to question why we have changed opinion on the feasibility of the electric. Have we really? And should we really?

Keywords : automobilism, automobile culture, car technology, electric automobile.

En mai 2011, l'Allemagne a célébré les «125 ans de l'automobile», en mettant en valeur ses pères fondateurs, Karl Benz et Gottlieb Daimler. Dans le même temps, la ville de Stuttgart, où ont eu lieu les festivités, a non seulement voulu regarder en arrière, mais aussi vers l'avenir en vantant la voiture électrique comme fondement des politiques locales et régionales pour l'amélioration de la «mobilité durable». Ironiquement, si la ville de Stuttgart et l'Allemagne sont amenées à avoir du succès en la matière, l'histoire de l'automobile nécessitera une réécriture au détriment de Benz et Daimler qui devront être détrônés: dès 1881 , soit cinq ans avant les deux illustres Allemands, un Parisien, le constructeur de calèche Charles Jeantaud, avait équipé un véhicule de la propulsion électrique ${ }^{1}$. Mais, même à ce compte, la question de savoir qui a inventé «l'automobile» ne sera pas définitivement résolue car elle ne peut pas l'être. Si jamais, en effet, nous décidions de mettre nos espoirs dans la voiture à vapeur comme devant être la voiture de l'avenir, alors nous célébrerions bientôt un bicentenaire, étant donné que les premières expériences sérieuses avec des omnibus à vapeur ont eu lieu au Royaume-Uni, aux alentours des années 1820.

Indépendamment du résultat de cette compétition entre, d'une part, des systèmes de propulsion et, d'autre part, des pays, l'ouverture de la perspective aux trois filières de propulsion classiques, remises à plat, permet de renouveler l'historiographie de la mobilité routière, comme elle résout la question de savoir pourquoi nous n'avons pas opté beaucoup plus tôt pour un récit centré sur l'alternative électrique. Dans cet article, la réponse à cette question sera culturelle plutôt que technique, soulignant le fait que la culture automobile dominante a très tôt défini la voiture comme devant être dotée d'un moteur à combustion interne. Il reste qu'expliquer l'échec du véhicule électrique par la victoire du pétrole est plus tautologique que fécond. Cependant, nous questionnerons simultanément dans ces pages les éléments techniques et culturels qui ont fait de l'alternative électrique une sempiternelle «auto de demain», si bien qu'à la fin nous resterons confrontés à cette question ultime: peut-elle devenir l'automobile d'aujourd'hui? 


\section{La première génération (1881-1902)}

La conviction de bon sens qui veut que la voiture électrique a un défaut technique rédhibitoire (le manque d'autonomie) repose sur une analyse imparfaite de l'échec de la première génération, qui vit les calèches électrifiées alors surtout utilisées dans des flottes urbaines de taxis. En fait, la domination souvent avancée du véhicule électrique parmi les trois filières de propulsion aux États-Unis peu avant 1900 n'était presque entièrement basée que sur cet usage professionnel. Si nous comptons, en revanche, seulement les automobiles utilisées à titre privé, les voitures à vapeur, comparées aux voitures électriques, constituaient sans aucun doute la majorité du parc ${ }^{2}$. Si, toutefois, on avait interrogé les experts qui commençaient à motoriser, autour de 1898, quelques flottes de taxis à Londres, Paris et dans plusieurs villes américaines comme New York et Boston, la réponse aurait été claire: le véhicule électrique offrait de loin la technique la plus rentable. D'ailleurs, en témoigne aussi le fait qu'autour de 1900, il n'y avait pas une seule expérience de taxis à grande échelle fondée sur la technique du moteur à combustion, qu'elle ait été externe (à vapeur) ou interne (à essence).

Ces premières initiatives échouèrent toutes du fait de la conjonction de problèmes techniques et managériaux, aggravés aux États-Unis par les dégâts de la spéculation financière ${ }^{3}$. Mais le point de départ des ennuis était clairement technique: la masse active tombant des plaques dans les batteries au plomb causait des courts-circuits et contribuait à réduire leur longévité. À quoi il fallait ajouter l'usure rapide des pneumatiques incapables de porter le poids supplémentaire des batteries. Autrement dit, le contexte commercial de ces premières expériences à grande échelle de véhicules électriques leur fut néfaste, les augmentations de coûts menant bientôt à l'abandon des initiatives, tandis que, dans le même temps, les voitures à essence, quoique beaucoup moins fiables, bénéficiaient d'une utilisation par les membres d'une élite qui n'avaient pas à se soucier de la modicité des coûts, beaucoup d'entre eux possédant plusieurs voitures.

On peut toutefois s'interroger pour savoir pourquoi aussi peu de conducteurs potentiels choisirent une technologie pourtant bien plus fiable. Mis à part le refus de s'engager dans la maintenance de leur batterie, un exercice assimilable à une chimie de laboratoire, ces premiers utilisateurs privés avaient concomitamment développé une culture dominée par l'aventure automobile, la course et le tourisme, mais aussi par le goût du bricolage nécessaire pour surmonter les pannes, à l'image du comportement des premiers consommateurs de PC bien plus tard. Pour eux, les imperfections de la voiture à essence étaient une vertu plutôt qu'un problème, une source de plaisir et de relaxation, à l'image du jardinage, comme l'un d'entre eux l'a déclaré. Ces utilisateurs masculins n'étaient pas intéressés par un véhicule qui, comme le britannique T. Chambers l'a consigné dans son journal en 1907, "fonctionne toujours». Il notait qu'«il n'y a pas beaucoup de sport dans la conduite d'un véhicule électrique. [...] Il est beaucoup trop peu intéressant pour être attractif. 
La fascination pour un moteur à essence chez un homme né avec un instinct d'ingénierie, provient de ses imperfections et de ses excentricités ${ }^{4}$.»

Et pourtant, bien que les premières expériences des années suivantes aient suscité chez les entrepreneurs beaucoup d'hésitations quant à la motorisation de leurs flottes, ces tentatives fournissent des enseignements intéressants qui ont malheureusement pour la plupart été oubliés. L'un d'eux tient à la recharge de la batterie pour le ravitaillement en énergie pratiquée aux États-Unis: à New York, la Société du véhicule électrique avait installé une station de recharge sur Broadway, où les systèmes pouvaient être échangés semi-automatiquement en environ soixante-dix secondes, soit plus rapidement que pour le remplissage d'un réservoir d'essence. De la même façon, en France, une proposition avait été lancée pour permettre "un Tour de France électrique» avec des stations pour les échanges de batteries, une proposition qui avait eu une suite, également avortée, aux Pays-Bas. Ces expériences témoignent du fait que l'argument de l'autonomie n'est pas valable: si nous l'avions voulu, nous aurions pu dis- poser tous les cinquante kilomètres d'un système d'échange standard de batteries, ainsi que le propose de nos jours la société Better Place ${ }^{5}$.

Un deuxième enseignement de cette première période tient au fait que nous devrions être prudents quant à la comparaison des contextes d'usage des automobiles électriques et des voitures à combustion. Pour des taxis ou des véhicules privés, les contextes d'utilisation sont tout à fait différents, comme le sont les usages des voitures de course et ceux des voitures d'usage courant. La Jamais Contente, voiture électrique avec laquelle le pilote Camille Jenatzy a battu le record de vitesse des $100 \mathrm{~km} / \mathrm{h}$ en 1899, n'avait pas eu à couvrir plus d'un kilomètre. A contrario, les Grands Prix ultérieurs et les autres concours de vitesse ont été entièrement dominés par les voitures à essence. L'électricité ne cadrait donc pas avec le monde de la course, non pas parce qu'elle ne fonctionnait pas correctement, mais parce que la culture de course a été façonnée sur le modèle du moteur à pétrole. Le succès de la Jamais Contente démontre donc que l'idée de vitesse dépend de son contexte, de la période et du lieu de son application.

\section{La deuxième génération (1902-1925)}

À première vue, la deuxième génération de véhicules électriques routiers ne différait pas beaucoup de la première: de façon similaire, des automobiles déjà passées de mode aux yeux des contemporains eux-mêmes furent utilisées dans des flottes de taxis en Allemagne et aux Pays-Bas, ainsi que dans plusieurs flottes urbaines de voitures de pompiers en Allemagne. Mais les batteries et les techniques pneumatiques s'étaient entretemps considérablement améliorées. Là ne résidait cependant pas la différence cruciale. Tout autour de ces techniques éprouvées, un nouveau business model avait été bâti pour pallier les éventuelles 


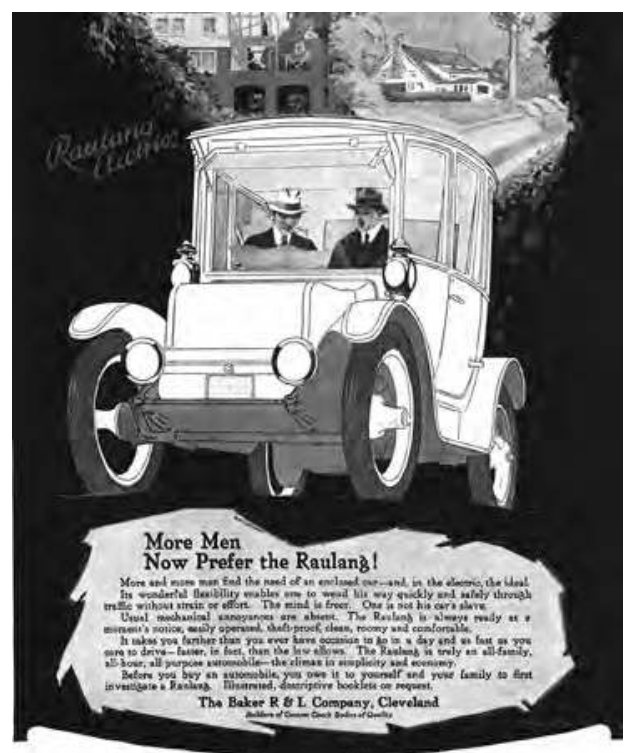

Fig. 1. Publicité pour la Raulang électrique à la fin des années 1910 (Coll. part.).

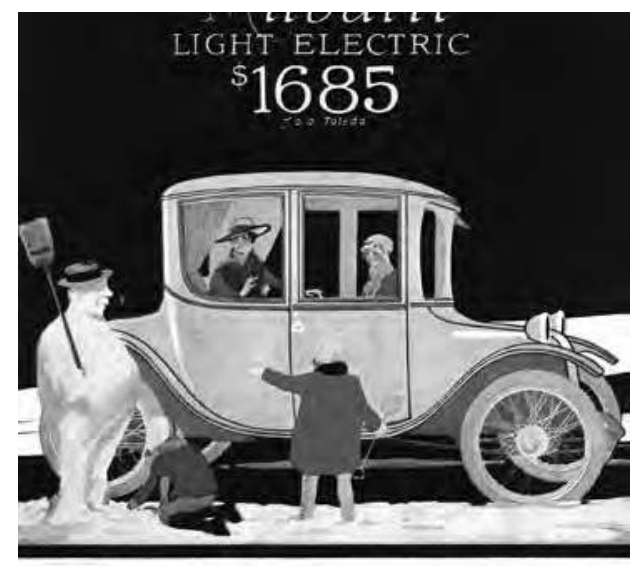

Greater Beauty-Greater Dependability

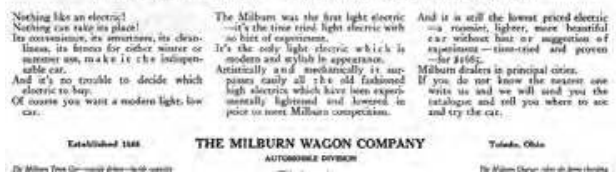

Fig. 2. Publicité pour la Milburn électrique au milieu des années 1910 (Coll. part.).

ture de maintenance centralisée, bien triques furent menées à bien avec le même modèle franco-allemand de taxis (Kriéger-Namag), appuyé solidement sur un garage central où des techniciens spécialisés échangeaient les batteries défectueuses, ainsi que les trains de pneus quand cela était nécessaire. Pour une modeste somme de quelques cents ou pfennig par kilomètre parcouru dans le cas d'Amsterdam (les flottes allemandes ayant été abandonnées à cause de la guerre), on obtenait des prestations supérieures à celles qui étaient fournies par les alternatives à essence, simultanément mises en place.

Dans certains cas limites expérimentaux (flottes de taxi minuscules, comme un taxi conduit par un père et son fils), la propulsion à essence avait une chance: pour ce type d'applications, il n'était pas réaliste d'investir dans une infrastrucque, s'il avait existé à ce moment-là un réseau général de stations de recharge, de telles initiatives de taxis auraient aussi bien pu s'en servir. En réalité, avant la Première Guerre mondiale, une utilisation coopérative de stations centralisées se produisit à Berlin pendant une courte période. De même, les pompiers allemands étaient-ils satisfaits de leurs véhicules électriques (et même hybrides, car certains d'entre eux étaient à vapeur-électriques!). Ces flottes de véhicules de six tonnes nous conduisent à un troisième enseignement que nous pouvons tirer de cette histoire: l'idée que la traction électrique ne peut pas faire face à de lourdes charges doit être abandonnée depuis que le chef du corps des pompiers de Berlin, Maximilian Reichel, pourtant supporter de la vapeur, se déclara en faveur de la traction élec- 
trique pour son projet de motorisation, bientôt suivi par ses homologues dans d'autres villes allemandes ${ }^{6}$. Là, l'alternative des véhicules à essence s'est faufilée dans les marges, notamment dans les petites villes qui ne disposaient que d'un ou deux véhicules.

Un quatrième enseignement est encore plus important: les initiatives des taxis de seconde génération montrent clairement que la technologie seule ne peut jamais être un argument convaincant pour expliquer l'échec. En assurant une fiabilité minimale, de tels défauts pouvaient toujours être corrigés par un surcroît d'organisation ${ }^{7}$. Ceci, néanmoins, est seulement vrai pour des exploitations en flottes. En revanche, pour garer son véhicule, un propriétaire individuel devait avoir recours au réseau de garages, alors en cours de constitution, où l'entretien était simultanément assuré par un personnel spécialisé qui prenait ainsi en charge cet aspect de la culture automobile. Mais cette pratique a été bientôt abandonnée en faveur d'une transformation de la remise des autos (à pétrole), où celles-ci demeuraient toujours à proximité et à disposition, pouvant être prises sans beaucoup de préparation.

La Première Guerre mondiale a joué un rôle décisif dans ce développement général, à cause de la préférence claire des militaires pour l'alternative à essence. Pendant la guerre, des installations de production massives furent établies, mais également des milliers de jeunes hommes reçurent une formation à la conduite des camions à essence. Ces éléments fournirent la base pour le boom de l'après-guerre, qui se traduisit par une explosion de la motorisation petite bourgeoise et rendit l'alternative électrique désuète. Ceci fut particulièrement le cas aux États-Unis où, pendant la guerre, une vague d'utilisation de véhicules électriques a semblé se lever. Mais le parc d'environ 30000 voitures électriques particulières qui, avec environ 10000 camions électriques, a constitué la présence la plus importante d'une culture électrique de l'automobile pendant la première moitié du $x^{e}$ siècle, a rapidement disparu. En principe, le «tourisme électrique», comme on l'appelait alors, était même possible, ainsi qu'en témoigne une carte du réseau des stations de recharge autour de New York. En attendant, la technique rivale avait pris le contrôle sur tant d'avantages par rapport à l'électricité qu'il était devenu de plus en plus difficile de dévier du modèle de culture automobile dominante Une grande partie de cette culture était toujours axée sur le plaisir et commençait à se développer dans le domaine du tourisme à longue distance ${ }^{8}$.

Ceci est le cinquième enseignement que je voudrais tirer de cette histoire: une fois qu' elle est dominante, une technique essaie d'absorber tous les avantages des techniques concurrentes pour les rendre plus difficiles à utiliser pour le consommateur. J'ai appelé ce mécanisme l'effet Pluto: le chien de Walt Disney (jouant le rôle de l'ingénieur de la technique alternative) court après une saucisse tenue devant son nez, le bâton tenant la saucisse étant dirigé par l'homme sur le chariot (représentant la technique dominante). Il conduit ainsi l'innovation, mais ne semble pas, ce faisant, atteindre son propre but. Cela s'applique à la compétition entre essence et électricité: les 
partisans de l'essence ont non seulement repris les carrosseries à conduite intérieure, le démarrage automatique et le roulage silencieux de l'unité de propulsion de l'électrique, mais ils ont aussi intégré dans leur paradigme les pneumatiques endurants, à l'origine développés pour les poids lourds électriques, et l'on peut même soutenir que la maintenance des véhicules contre les pannes au moyen d'une infrastructure d'assistance fiable et l'idée générale de «service» ont été inspirées des premières expériences de véhicules électriques? .

\section{La troisième génération et au-delà}

Les fabricants de véhicules électriques n'ont jamais investi beaucoup de temps ni d'argent dans la conception de leur propre forme de carrosserie. Les originalités sont rares. La Jamais Contente du belge Jenatzy, avec son pseudo aérodynamisme, dépassa pour la première fois les $100 \mathrm{~km} / \mathrm{h}$. Les taxis Kriéger, avec quelques produits du leader du marché américain, Detroit Electric, suggérèrent une «voiture de ville», appellation qui, pendant le demi-siècle suivant, resta lettre-morte jusqu'à ce que les constructeurs d'automobiles à essence la redécouvrent après la Seconde Guerre mondiale. Jusqu'à ce moment et pour longtemps, l'automobile électrique s'est glissée dans les formes du design courant des carrosseries des voitures à essence, comme si elle avait voulu cacher sa différence. Kriéger lui-même, pendant la Seconde Guerre mondiale, avait conçu une carrosserie se présentant comme une potentielle voiture à essence, dans laquelle un capot cachait les batteries ${ }^{10}$. Il n'en reste pas moins que la conduite en voiture électrique a littéralement disparu de l'espace public et a trouvé une niche dans l'usage industriel des chariots de manutention, dont des milliers d'exemplaires ont été construits pen- dant les années d'entre-deux-guerres. La culture utilitariste du camion électrique se diffusa particulièrement en Allemagne, sous l'impulsion du géant des piles AFA, plus tard Varta; mais c'est au Royaume-Uni, pendant les années 1930, que la plus grande flotte jamais développée a été mise en circulation, sous la forme de dizaines de milliers de camionnettes électriques de livraison de lait. Elles firent de ce pays le plus important dans le monde pour l'automobilisme électrique pendant deux décennies après la Seconde Guerre mondiale ${ }^{11}$.

Dans cette perspective, on peut se demander s'il est digne d'intérêt de distinguer, après la Seconde Guerre mondiale, une nouvelle génération d'expériences de véhicules électriques pour ceux qui sont apparus pendant les années 1960, bien avant les crises de l'énergie des années 1970 qui seraient à l'origine de ce qui est généralement considéré comme la génération suivante. La plupart de ces véhicules (camions et utilitaires légers), développés avec une préoccupation environnementale et un souci d'aménité urbaine (et aussi parce que, dans la phase immédiate de l'après-guerre, tout semblait techniquement possible), étaient bien plutôt des 
conversions. On voyait souvent de telles re-configurations de designs de véhicules existants comme des laboratoires utilisant de nouveaux types de moteurs électriques et, plus spécifiquement, de nouveaux types de batteries, avec de préférence des variantes de haute énergie comme le zinc-air ou le sodium-soufre ${ }^{12}$.

Il y a, cependant, au moins un argument pour parler d'une nouvelle génération, du fait de l'apparition d'une nouvelle espèce de voitures, à savoir des automobiles urbaines minuscules, construites par de petites sociétés qui désiraient démontrer que les grands constructeurs avaient tout faux. Alors, peut-être comme une réponse à cette dizaine d'initiatives, les constructeurs automobiles établis commencèrent à proposer des prototypes entièrement nouveaux. L'une des plus controversées fut la Smart, parce qu'elle avait été, à l'origine, conçue comme une pure voiture électrique urbaine par un fabricant de montres suisse. Une fois reprise par Daimler-Benz, elle fut équipée d'un moteur à essence, signe du fait que l'automobilisme électrique dépendait des préférences constamment changeantes des fabricants, des gouvernements et des propriétaires de flottes et de l'hésitation constante des grands constructeurs automobiles devant la taille future du marché automobile électrique ${ }^{13}$.

Il y a une autre raison pour laquelle il semble justifié de distinguer plusieurs vagues dans la pratique des voitures électriques au cours de l'après Seconde Guerre mondiale. Si une génération est identifiable dans les années 1970, encouragée par la recherche d'une batterie miracle, la génération suivante commença en 1990, après une période d'accalmie pendant les années 1980, avec la fondation du California Air Ressource Board (CARB). Ses décisions ont visé à introduire massivement la conduite électrique chez les citoyens de cet État ${ }^{14}$. En 2003, ses responsables prescrivirent qu'un dixième des véhicules des grandes sociétés vendus en Californie devrait être «zéro émission» (ZEV). Inspirées par cette initiative, des expériences à plus petite échelle eurent lieu en Europe, comme dans l'île allemande de Rügen (toujours conduites par l'espoir de la découverte d'une batterie haute capacité par les principaux fabricants) ou dans les villes de La Rochelle en France et de Mendrisio en Suisse. Dans les deux cas, l'usager final était visé par les grands constructeurs (PSA pour La Rochelle) ou des petites sociétés.

Toutefois, il y a aussi beaucoup de ressemblances avec les développements de véhicules électriques réalisés avantguerre. La plus remarquable continuité, qui s'intensifie alors, est la quête d'une batterie miracle, déjà engagée au début des années 1900, lorsque Thomas Edison avait travaillé sur sa batterie «d'acier», en nickel-fer, promettant une capacité d'énergie plus élevée (et donc plus de kilomètres de conduite pour un kilogramme donné de batterie) et un rechargement plus rapide sans recours à l'échange de batterie. Depuis lors, nous avons vu que le cadmium-nickel est réapparu (pour disparaître de nouveau), suivi par la pile à combustible (puisqu'au début des années 1960, des applications spatiales avaient eu lieu) et également, dans une moindre mesure, une douzaine d'autres variantes moins exotiques, comme le nickel-métal-hybride au gel sans maintenance, et, plus récemment, les combinaisons lithium-ion. 
Ces différents essais peuvent se lire comme le reflet de l'incertitude des principaux acteurs quant aux réelles raisons $\mathrm{du}$ manque d'enthousiasme des automobilistes pour la voiture électrique. Comme les conversions techniques, le public aurait aussi dû être converti, ce que plusieurs projets introduits par des spécialistes des sciences humaines ont essayé de faire: une fois expérimenté le nouveau style de conduite électrique, le public aurait dû développer une attitude plus écologiquement consciente et plus rationnelle quant à l'utilisation de la voiture $^{15}$. Cette idée que le grand public aurait été délibérément éloigné de cette forme vertueuse de transport, s'est aussi étendue chez les défenseurs du mouvement électrique. Ceci a, par exemple, été démontré avec une grande éloquence par le film Qui a tué la voiture électrique? et sa suite, La vengeance de la voiture électrique, par le même réalisateur ${ }^{16}$.

Bien que l'on n'ait pas besoin de recourir à une théorie du complot pour expliquer la chaîne d'événements constituant l'histoire de la voiture électrique au $\mathrm{xx}^{\mathrm{e}}$ siècle, le comportement de General Motors, pris de panique et éliminant son EV1 malgré son succès relatif, et les actions de lobbying caché et les campagnes publiques des compagnies pétrolières pour obtenir l'abandon des décisions du CARB promouvant le $\mathrm{ZEV}$, démontrent qu'à cette occasion, en 2003, un réel tournant a presque été atteint. Il fut suivi par une frustration profonde de beaucoup des parties prenantes désillusionnées, qui s'orientèrent par la suite souvent vers des hybrides, parti- culièrement après le lancement réussi de la Toyota Prius, dont deux millions d'exemplaires furent vendus à la fin du siècle dernier ${ }^{17}$.

Ceci est une deuxième preuve de la continuité de l'histoire de la voiture électrique depuis le siècle passé. Comme la batterie miracle, l'hybride est aussi vieux que l'option électrique. Il a fonctionné et évolue comme un véhicule de transition entre deux paradigmes: la combustion interne contre l'électricité. Au prix d'une complexité supplémentaire (qui semble depuis être gérable avec l'électronique moderne), l'hybride permet de rouler avec l'électricité en ville et à la vitesse pleine sur les routes et autoroutes. Ironiquement, l'hybride interroge donc le tout dernier argument solide avancé en faveur de la propulsion électrique pure: éviter la pollution locale dans les quartiers les plus exposés. Cet objectif semble être mieux rempli par l'électrification des nombreuses camionnettes de livraison avec leur système de mise en veille pour gérer les fréquents arrêts et redémarrages. En revanche, ces efforts d'électrification des deuxièmes voitures pour les ménages, qui aboutissent à des voitures urbaines drôlement stylisées pour la ville, contribuent non pas à réduire la pollution locale, mais à augmenter l'espace urbain dévoré par l'automobile.

La question est alors de savoir si nous voulons toujours promouvoir l'automobilisme électrique, alors que tout le monde sait maintenant que la clef pour diminuer la contribution de la voiture au changement et au réchauffement climatique n'est pas un changement de moteur. 


\section{L'effet Pluto}

Il est d'autant plus difficile de répondre à cette question que l'effet Pluto a minimisé les avantages des véhicules électriques en engageant un mouvement qui a continuellement réduit l'écart entre les deux techniques rivales: les calculs économiques du rendement de la source d'énergie au volant sont au mieux ambigus et ne semblent pas indiquer d'avantage évident pour l'alternative électrique, à moins que l'on ne vive dans des pays ayant beaucoup d'énergie nucléaire, comme la France, ou de houille blanche, comme la Norvège.

Il n'y a aucun doute que, quand on est intéresséà réduire la pollution locale pour combattre le smog et les particules fines, l'énergie électrique n'a aucun égal (bien que nous ayons vu que cela puisse être contesté par un hybride bien conçu). La répugnance, que l'on trouve également chez certains spécialistes des sciences humaines, pour analyser sérieusement le pourquoi de l'échec des expériences les plus récentes et apparemment très prometteuses des années 1990, est révélatrice: sommes-nous vraiment enclins à apprendre des erreurs du passé, étant donnée l'ardeur avec laquelle les parties prenantes sont passées à l'expérience suivante comme si elles craignaient que, regardant derrière elles, hier ne nous rapproche pas de demain? Les historiens savent, eux, que l'opposé pourrait bien être plus proche de la vérité.

Indépendamment $\mathrm{du}$ résultat de telles analyses, qu'il convient de toujours améliorer, il est tout à fait clair que le rôle historique majeur du véhicule électrique, aux côtés de plusieurs autres alternatives sérieuses au moteur à essence à com- bustion interne, comme la turbine à gaz dans les années 1960, ou la combustion interne avec les carburants alternatifs comme le gaz naturel ou le biodiesel, a été d'inciter et même de forcer la technologie hégémonique à accélérer son évolution technique et organisationnelle de telle façon qu'à la fin, la prime à l'adoption d'une autre technique était considérablement affaiblie. Comme des myopes, nous regardons fixement l'artefact carrossé (qui pourrait être remplacé par un autre), tandis que c'est sous le capot, plus par évolutions que par révolutions, que la plupart des changements sont advenus et continueront de se faire. Ce qui cache aussi le fait que, peut-être, nous ne sommes pas du tout intéressés par ce changement, et que nous voudrions garder la «voiture de demain» comme un alibi pour continuer à faire ce que nous (les hommes? les femmes aussi?) aimons secrètement faire: rouler dans une voiture à combustion, rapide, pleine de vibrations, éventuellement électrifiée, convertie au gaz naturel, aux biocarburants ou même à l'hydrogène lorsque nous serons à court de pétrole.

\section{Notes}

1. Gijs Mom, The electric vehicle. Technology and expectations in the automobile age, Baltimore, Johns Hopkins University Press, 2004, p. 17; les pages qui suivent se fondent pour l'essentiel sur cet ouvrage. Voir également David A. Kirsch, The electric vehicle and the burden of history, New Brunswick, New Jersey/Londres, Rutgers University Press, 2000, et Dietmar Abt, Die Erklärung der Technikgenese des Elektroautomobils, Francfort/Berlin/ Berne/New York/Paris/Vienne, Peter Lang, 1998.

2. Cette confusion est très présente dans les études où l'analyse historique n'est que de seconde main: René Kemp, Frank W. Geels, Geoff 
Dudley, «Introduction: sustainable transitions in the automobility regime and the need for a new perspective», dans Frank W. Geels, René Kemp, Geoff Dudley, Glenn Lyons (éd.), Automobility in transition? A socio-technical analysis of sustainable transport, New York/Londres, Routledge, 2012, p. 3-28, ici p. 23.

3. Pour une analyse de ces expériences précoces, D. A. Kirsch, The electric vehicle and the burden of history, op. cit.

4. Cité par Montagu of Beaulieu et F. Wilson Combs, Behind the wheel: the magic and manners of early motoring, New York, Paddington Press, 1977, p. 111-112.

5. Renato J. Orsato, Marc Dijk, René Kemp, Masaru Yarime, "The electrification of automobility: the bumpy ride of electric vehicles toward regime transition", dans F. W. Geels et al. (éd.), Automobility in transition?..., op. cit., p. 205-228, ici p. 214-215.

6. Gijs Mom, «Wie Feuer und Wasser: der Kampf um den Fahrzeugantrieb bei der deutschen Feuerwehr (1900-1940)», dans Harry Niemann, Armin Hermann (éd.), 100 jahre LKW. Geschichte und Zukunft des Nutzfahrzeuges, Stuttgart, Franz Steiner Verlag, 1997, p. 263-320.

7. Et depuis les ingénieurs se plaignent: «God does not want us to have full-function electric vehicles» (G. Mom, The electric vehicle, op. cit., p. 275). Cette opinion, avancée dans une audition par le Congrès d'un responsable de Ford en 2000, est parfois relayée par des chercheurs en sciences sociales qui font confiance aux jugements des ingénieurs comme, par exemple, R. J. Orsato et al., "The electrification of automobility...», op. cit., p. 209, qui soulignent «les limites du progrès technique» comme étant le principal facteur de l'échec des expérimentations californiennes des années 1990. Pour une critique de ce better battery bugaboo, Michael Brian Schiffer, Taking charge: the electric automobile in America, Washington/Londres, Smithsonian Institution Press, 1994, p. 183.
8. Gijs Mom, Atlantic automobilism: emergence and persistence of the car in Europe and the USA, 1895-1940, New York, Berghahn, 2014.

9. Pour une lecture approfondie de ces processus, Gijs Mom, «Translating properties into functions (and vice versa): design, user culture and the creation of an American and a European car (1930-1970)», Journal of design history, vol. 20, n², 2007, p. 171-181.

10. Pascal Griset, Dominique Larroque, L'odyssée du transport électrique, Paris, Cliomédia, 2006, p. 98.

11. Dietmar Abt, Die Erklärung der Technikgenese des Elektroautomobils, op. cit.

12. Remigius Johannes Franciscus Hoogma, Exploiting technological niches. Strategies for experimental introduction of electric vehicles, Enschede, Twente University Press, 2000.

13. Roland Wolf, Le véhicule électrique gagne le cœur de la ville, Paris, Centre français de l'électricité, 1999 , p. 318.

14. Daniel Sperling, Future drive : electric vehicles and sustainable transportation, Covelo, Island Press, 1995 , p. 27; Mark B. Brown, «The civic shaping of technology: California's electric vehicle program ", Science, technology $\mathcal{E}$ human values, vol. 26, $\mathrm{n}^{\circ} 1$ (Winter 2001), p. 56-81.

15. Heide Gjøen, Michael Hård, «Cultural politics in action: developing user scripts in relation to the electric vehicle», Science, technology $\mathcal{E}$ human values, vol. 27, $\mathrm{n}^{\circ} 2$ (Spring 2002), p. 262-281.

16. Who killed the electric car? (Sony Pictures Classics, 2006, dir. Chris Paine); Revenge of the electric car (West Mid Westr Productions and Area 23A, 2011, dir. Chris Paine).

17. Bruce Pietrykowski, «The curious popularity of the Toyota Prius in the United States ", dans Weert Canzler, Gert Schmidt (éd.), Zukünfte des automobils: aussichten und grenzen der autotechnischen Globalisierung, Berlin, Sigma, 2008, p. 199211. 